\title{
CONTEXTO DE VIDA DE PESSOAS USUÁRIAS DE DROGAS NA PERSPECTIVA DE GÊNERO
}

LIFE CONTEXT OF PEOPLE WHOM ARE DRUG USERS ON GENDER PERSPECTIVE

Michele Mandagará Oliveira, Valéria Cristina Christello Coimbra, Roberta Zaffalon Ferreira, Poliana Farias Alves, Karine Langmantel Silveira, Paola de Oliveira Camargo, Gabriela Lanzetta Haack, Gabriela Botelho Pereira, Suelen Cardoso Leite

\section{RESUMO}

Objetivo: Caracterizar o contexto de vida e vulnerabilidade de pessoas usuárias de drogas na perspectiva do gênero. Método: Estudo transversal exploratório com 505 usuários de drogas. Foram consideradas as variáveis: gênero, perfil socioeconômico e eventos ocorridos no último ano. Resultados: Observou-se que mulheres usuárias de drogas, mesmo em menor número do que os homens, apresentaram maior ocorrência de violência sofrida e abuso sexual, perda de seus filhos para o conselho tutelar e mudanças em seus hábitos de vida, como o sono. Conclusões: É necessário refletir a respeito do desenvolvimento de políticas públicas sobre a atenção oferecida para as pessoas que considerando a perspectiva de gênero.

Descritores: Gênero e saúde; Cocaína crack; Vulnerabilidade social; Saúde mental.

\section{ABSTRACT}

Objective: This study aimed to characterize the life context and the vulnerability of drug users by the gender perspective. Methodology: It is a cross sectional study, with 505 drug users. There were considered the variables: gender, socioeconomic profile and occurred events last year. Results: Women drug users, even if in lower number than men drug users, showed more violence suffered and sexual abuse, as well as child loss to the Child Protection Agency and changes in her habits, as example, the sleeping. Conclusions: It is necessary to reflect on the development of public policies on the care provided for people considering the gender perspective.

Descriptors: Gender and health; Crack cocaine; Social vulnerability; Mental health.

Michele Mandagará de Oliveira, Doutora em Enfermagem pela Universidade de São Paulo (USP), Ribeirão Preto, $\mathrm{SP}$, Brasil.

Valéria Cristina Chistello Coimbra, Doutora em Enfermagem pela Universidade de São Paulo (USP), Ribeirão Preto, SP, Brasil.

Roberta Zaffalon Ferreira, Doutoranda em Enfermagem da Universidade Federal de Pelotas (UFPel), Pelotas, RS, Brasil.

Poliana Farias Alves, Doutoranda em Enfermagem da Universidade Federal de Pelotas (UFPel), Pelotas, RS, Brasil.

Karine Langmantel Silveira, Mestranda em Enfermagem da Universidade Federal de Pelotas (UFPel), Pelotas, RS, Brasil.

Paola de Oliveira Camargo, Doutoranda em Enfermagem da Universidade Federal de Pelotas (UFPel), Pelotas, RS, Brasil.

Gabriela Lanzetta Haack, Mestranda em Enfermagem da Universidade Federal de Pelotas (UFPel), Pelotas, RS, Brasil.

Gabriela Botelho Pereira, Mestranda em Enfermagem da Universidade Federal de Pelotas (UFPel), Pelotas, RS, Brasil.

Suélen Cardoso Leita, Doutoranda em Enfermagem da Universidade Federal de Pelotas (UFPel), Pelotas, RS, Brasil. 
O uso abusivo de substâncias psicoativas no cenário nacional tem preocupado cada vez mais todas as esferas da sociedade, que é agravado pela inadequação de políticas que respeitem as necessidades sociais e de saúde dos usuários de forma integral e humanizada, com respeito e segurança. ${ }^{1}$ As políticas sociais e econômicas estão em contínua transformação, assim como o perfil dos usuários de substâncias psicoativas, que estão em constante mudança. Em pesquisa quantitativa, realizada entre os anos de 2011 e 2013, em território nacional, pela Fiocruz, identificou-se que no Brasil, os usuários de crack nas cenas de uso são predominantemente do gênero masculino - $78,7 \%$, embora haja um número cada vez maior de mulheres usuárias. ${ }^{2}$

Pensando no cuidado oferecido aos usuários de substâncias psicoativas durante décadas, o mesmo era prestado sob a lógica manicomial, com abordagens moralistas e reducionistas que não levavam em conta as especificidades e outros fatores que envolvem o uso de drogas. ${ }^{3}$ Buscando superar 0 atraso histórico relacionado às políticas de saúde pública voltadas aos usuários de substâncias psicoativas, o Ministério da Saúde em 2003, definiu a Política de Atenção Integral ao Usuário de Álcool e Outras Drogas com vistas a garantir o cuidado integral e enfrentar os diferentes problemas relacionados ao consumo de substâncias. ${ }^{4}$

O Ministério da Saúde tem buscado, em conjunto com outras políticas sociais, implementar e reforçar a qualidade do atendimento aos usuários por meio de serviços substitutivos ao modelo manicomial, como o Centros de Atenção Psicossocial Álcool e Drogas (CAPS AD) e os Serviços de Redução de Danos (RD), objetivando uma assistência integral articulada em rede e em todos os níveis de atenção em saúde, fortalecendo o cuidado extra hospitalar ${ }^{4}$

Durante muito tempo 0 uso de substâncias psicoativas era considerado apenas um problema do universo masculino. Pensando nas pesquisas sobre o uso de substâncias psicoativas relacionadas às questões de gênero, importante ressaltar que as diferenças entre homens e mulheres emergem também do impacto sociocultural existente dentro das construções de feminilidade e masculinidade sobre 0 uso, visto que as questões de gênero são permeadas por costumes e valores que determinam os papéis que cada um assume, havendo ligação direta com a constituição de subjetividade e, portanto, com a forma de se ver e estar no mundo. ${ }^{5}$

As desigualdades em questões de gênero pautados nas distinções entre homens e mulheres estão presentes em diversos aspectos no que diz respeito ao consumo de drogas, tais como prevalência, metabolismo $e$ fisiologia, mortalidade, tratamento, vitimização, violência, comorbidades entre outros. ${ }^{6}$

Outra diferença encontrada aparece nas relações e vínculos existentes, enquanto os homens têm mais problemas profissionais e legais, como 0 envolvimento com roubo e tráfico, as mulheres têm mais problemas físicos e familiares, agravados por fatores de risco como uso do corpo para obter droga, prostituição e infecções sexualmente transmissiveis ${ }^{\circ}$

Diante do exposto, o presente artigo teve como objetivo caracterizar o contexto de vida e a vulnerabilidade de homens e mulheres assistidos pelos serviços de CAPS AD e Redução de Danos de um município do extremo sul do Brasil na perspectiva de gênero.

Metodologia

Este estudo é parte integrante do projeto de pesquisa "Perfil dos Usuários de Crack e Padrões de Uso", que foi financiado pelo Conselho Nacional de Desenvolvimento Científico e Tecnológico (CNPq), pelo edital MCT/CNPq n 041/2010. Trata-se de um estudo de corte transversal e exploratório.

Foi obtida uma amostra estratificada dos serviços de RD e CAPS AD, com o objetivo de estimar a proporção de usuários de drogas no município. Para o cálculo, utilizaram-se as informações fornecidas pelo sistema de informação dos serviços. A prevalência de usuários de drogas foi desconhecida $(p=0,50)$, admitiu-se um erro amostral de $4 \%$ $(\mathrm{d}=0,04)$, sob o nível de confiança de $95 \%(a=0,05)$, no denominador adotou-se o total de indivíduos cadastrados do RD $(\mathrm{N}=5.700)$ e CAPS AD ( $\mathrm{N}=200)$. O n encontrado foi alocado proporcionalmente aos respectivos estratos e acrescentouse 10\% para substituição de perdas eventuais. Ao final se obteve uma amostra de 505 entrevistas, e deste total, 436 sujeitos pertenciam à estratégia RD e 69 ao CAPS AD. A sistemática de seleção adotada foi o sorteio direto nas bases de dados dos respectivos serviços.

Foi realizado um teste piloto com 14 usuários indicados pela estratégia de Redução de Danos, visando testar o instrumento, operacionalizar a coleta de dados no campo e avaliação das práticas dos entrevistadores. Os dados foram coletados mediante entrevistas por meio de um questionário estruturado, e após o retorno do campo, os questionários foram codificados pelo entrevistador e revisados pelos coordenadores de campo.

Os dados foram digitados utilizando o gerenciador de banco Microsoft Access v.2003 e após a digitação, foram exportados para o software estatístico STATA v.12 para geração dos resultados e gráficos. 0 controle de qualidade dos dados foi realizado em três etapas distintas: supervisão de campo, supervisão da codificação dos dados e replicação de $5 \%$ dos questionários válidos por meio de contato telefônico.

Para o presente estudo, foram selecionadas as seguintes variáveis: dependente (gênero dos participantes) e independentes (perfil socioeconômico e eventos ocorridos no último ano).

Os resultados foram apresentados mediante uso de distribuições de frequências uni e bi-variadas e medidas descritivas (médias e desvio padrão). As diferenças entre as proporções foram verificadas através dos Testes Quiquadrado de Pearson e de Tendência linear. Foi utilizada a prevalência como medida de ocorrência. Como medida de associação foi utilizada a Razão de Prevalência com intervalo de confiança a 95\%, obtida por meio da regressão de Poisson robusta, ao nível de $5 \%$ de significância estatística.

A pesquisa obedeceu aos princípios éticos da Resolução COFEN n 311/2007, Resoluções 196/96 e a 466/12 do Conselho Nacional de Saúde do Ministério da Saúde. O projeto foi aprovado pelo Comitê de Ética e Pesquisa da Faculdade de Enfermagem da Universidade Federal de Pelotas e recebeu o parecer n 301/2011.

RESULTADOS:

A população deste estudo foi constituída por 505 usuários de drogas assistidos pelos serviços de RD e CAPS AD. O gênero masculino foi predominante e representou $83,8 \%$ do total e o feminino $16,2 \%$. A média de idade foi de 38,8 anos (dp=13 anos), a mínima de 18 e a máxima de 76 anos. A distribuição etária revelou maior concentração de usuários adultos jovens com idade entre 20 a 39 anos $(52,3 \%)$. Com relação a cor auto referida, a branca foi predominante $(50,9 \%)$ seguido da preta $(22,0 \%)$, pardos e mestiços $(19,4 \%)$. Quanto ao estado civil, os solteiros e os casados/unidos predominaram na amostra $53,3 \%$ e $32,7 \%$, respectivamente (Tabela 1 ).

Tabela 1. Perfil sociodemográfico dos usuários de crack, álcool e outras drogas por gênero. Pelotas RS, Brasil, 2011-2012. $(n=505)$

\begin{tabular}{lrrrr}
\hline Característica & Total $\mathrm{n}(\%)$ & $\begin{array}{c}\text { Feminino 82 (16,2) } \\
\mathrm{n}(\%)\end{array}$ & $\begin{array}{c}\text { Masculino 423 (83,8) } \\
\mathrm{n}(\%)\end{array}$ & p-valor \\
\hline Grupo etário & & & & \\
$<20$ & $18(3,6)$ & $2(2,4)$ & $16(3,8)$ & $0,04 \mathrm{~b}$ \\
20 a 24 & $65(12,9)$ & $13(15,9)$ & $52(12,3)$ & \\
25 a 29 & $66(13,1)$ & $11(13,4)$ & $55(13,0)$ & \\
30 a 39 & $133(26,3)$ & $33(40,2)$ & $100(23,6)$ & \\
40 a 49 & $110(21,8)$ & $10(12,2)$ & $100(23,6)$ & \\
50 a 59 & $84(16,6)$ & $10(12,2)$ & $74(17,5)$ & \\
60 ou mais & $29(5,7)$ & $3(3,7)$ & $26(6,2)$ & \\
Cor da pele & & & & \\
Branca & $257(50,9)$ & $37(45,1)$ & $820(52,0)$ & \\
Parda/mestiça & $98(19,4)$ & $15(18,3)$ & $83(19,6)$ & \\
Preta & $111(22,0)$ & $24(29,3)$ & $33(7,8)$ & \\
Outra & $39(7,7)$ & $6(7,3)$ & $144(34,0)$ & \\
Situação conjugal & & & $217(51,3)$ & \\
Casado/com companheiro & $165(32,7)$ & $21(25,6)$ & $62(14,7)$ & \\
Solteiro & $269(53,3)$ & $52(63,4)$ & & \\
Divorciado/viúvo/separado & $71(14,0)$ & $9(11,0)$ & & \\
Escolaridade & & & & \\
& & & &
\end{tabular}




\begin{tabular}{lrrrr} 
Sem escolaridade & $10(2,0)$ & \multicolumn{1}{c}{$2(2,4)$} & $8(1,9)$ & $0,39 \mathrm{~b}$ \\
Fundamental incompleto & $327(64,7)$ & $52(63,4)$ & $275(65,0)$ & \\
Fundamental completo & $66(13,1)$ & $3(3,7)$ & $63(14,9)$ & \\
Ensino Médio incompleto & $45(8,9)$ & $10(12,2)$ & $35(8,3)$ & \\
Ensino Médio completo & $43(8,5)$ & $10(12,2)$ & $33(7,8)$ & \\
Superior incompleto & $8(1,6)$ & $1(1,2)$ & $7(1,6)$ & \\
Superior completo & $6(1,2)$ & $4(4,9)$ & $2(0,5)$ & \\
Situação de Atividade Econômica & & & & \\
Não & $147(29,1)$ & $13(15,9)$ & $105(24,8)$ & $0,279 a$ \\
Trabalho informal/eventual & $117(23,2)$ & $28(34,1)$ & $119(28,1)$ & \\
Trabalho formal & $118(23,3)$ & $22(26,8)$ & $95(22,5)$ & \\
Autônomo & $123(24,4)$ & $19(23,2)$ & $104(24,6)$ & \\
Renda Individual* & & & & \\
Sem renda & $51(10,1)$ & $14(17,1)$ & $37(8,7)$ & $0,00 \mathrm{~b}$ \\
< 1 salário & $175(34,6)$ & $46(56,1)$ & $129(30,5)$ & \\
1 até 2 salários & $224(44,4)$ & $16(19,5)$ & $208(49,2)$ & \\
Superior a 2 salários & $55(10,9)$ & $6(7,3)$ & $49(11,6)$ & \\
Renda Familiar* & & & & \\
Sem renda & $19(3,8)$ & $3(3,7)$ & $16(3,8)$ & $0,00 \mathrm{~b}$ \\
< 1 salário & $112(22,2)$ & $35(42,7)$ & $77(18,2)$ & \\
1 até 2 salários & $199(39,4)$ & $22(26,8)$ & $177(41,8)$ & \\
Superior 2 até 4 salários & $114(22,5)$ & $11(13,4)$ & $103(24,4)$ & \\
Superior a 4 salários & $48(9,5)$ & $9(11,0)$ & $39(9,2)$ & \\
Não souberam/não informaram & $13(2,6)$ & $2(2,4)$ & $11(2,6)$ & \\
\hline
\end{tabular}

Nota:*Salário mínimo R\$ 622,00; aTeste Qui-quadrado de Pearson;bTeste Qui-quadrado de Tendência Linear.

O perfil sociodemográfico das usuárias que utilizavam os serviços de CAPS AD e Redução de Danos se revelou proporcionalmente semelhante ao dos usuários e a amostra global, exceto para a distribuição das características grupo etário e rendimentos individual e familiar ( $p$-valor $>0,05$ )

As usuárias eram relativamente mais jovens quando comparadas aos usuários, 35,7 anos \pm 11 anos, mínima de 19 e máxima de 67 anos enquanto que, a média de idade dos usuários foi de 39,4 anos \pm 13 anos, mínima de 18 e máxima de 76 anos. Quanto à distribuicãa etária entre as usuárias, os grupos mais frequentes concentraram-se entre 30 a 39 anos $(40,0 \%)$ e entre 40 a 59 anos $(24,4 \%)$, as jovens representaram $29,3 \%$. Já entre os usuários, a distribuição etária entre os adultos de 30 a 39 e 40 a 59 anos foi semelhante $(23,6 \%$ ) e, os jovens representaram $25,3 \%$ e os $<20$ anos $3,8 \%$ e os idosos corresponderam a $6,8 \%$. Foram verificadas tendências proporcionais entre os grupos e estatisticamente significantes ( $p$-valor $=0,04)$

Os padrões de distribuição da cor e do estado civil foram semelhantes a amostra para ambos os grupos, se concentraram entre brancas e pretas e predominaram as solteiras $(63,4 \%)$ e os solteiros $(51,3 \%)$. Quanto aos níveis de escolaridade, a distribuição padrão foi semelhante ao global, $63,4 \%$ das usuárias possuíam o nível fundamental incompleto, entretanto eram mais escolarizadas quando comparadas aos usuários $(65,0 \%)$, houve uma maior frequência de usuárias com níveis de escolaridade entre o ensino médio e o superior.

Quanto à situação de atividade econômica, percebeu-se uma menor proporção de inativos entre as usuárias $(15.9 \%)$ quando comparadas aos usuários $(24,8 \%)$. As usuárias eram mais frequentes em atividades informais eventuais $(34,1 \%)$ quando comparadas aos usuários $(28,1 \%)$. Com relação ao rendimento individual, percebeu-se que as usuárias recebiam menos que os entrevistados do gênero masculino visto que, $73,2 \%$ das usuárias ganhavam menos que um salário mínimo quando comparadas aos usuários (60,8\%) que recebiam entre 1 a 2 salários mínimos ou superior. Quanto ao rendimento familiar, o declarado pelas usuárias entrevistadas foi inferior ao declarado pelos usuários, $42,7 \%$ das mulheres relataram rendimento $<1$ salário, enquanto $41,8 \%$ dos homens relataram que o rendimento familiar ficava entre 1 até 2 salários mínimos. Foram verificadas tendências proporcionais estatisticamente significantes entre a renda individual e renda familiar entre os grupos $(p-v a l o r<0,0001)$.
Os entrevistados foram questionados quanto às experiências e fatos importantes que ocorreram em suas vidas no último ano. Na Tabela 2, os eventos apresentam-se agregados segundo dimensões para a amostra total e estratificados por gênero.

Tabela 2. Eventos de vida ocorridos no último ano comparativo por gênero dos usuários de crack, álcool e outras drogas. Pelotas RS, Brasil, 2011-2012. ( $n=505)$

\begin{tabular}{|c|c|c|c|c|}
\hline Eventos ocorridos no último ano* & Total n (\%) & $\begin{array}{c}\text { Feminino } 82(16,2) \\
n(\%)\end{array}$ & $\begin{array}{c}\text { Masculino } 423(83,8) \\
\mathrm{n}(\%)\end{array}$ & $\mathrm{p}$-valora \\
\hline \multicolumn{5}{|l|}{ Família e relacionamentos } \\
\hline Casamento & $29(5,7)$ & $8(9,8)$ & $21(5,0)$ & 0,115 \\
\hline Divórcio ou separação & $78(15,5)$ & $15(18,3)$ & $63(14,9)$ & 0,409 \\
\hline Reconciliação com o companheiro & $48(9,5)$ & $11(13,4)$ & $37(8,7)$ & 0,215 \\
\hline Dificuldades Sexuais & $50(9,9)$ & $13(15,8)$ & $37(8,7)$ & 0,066 \\
\hline Perda de filho para o conselho & $13(2,6)$ & $5(6,1)$ & $8(1,9)$ & 0,044 \\
\hline Nascimento de criança na família & $163(32,3)$ & $38(46,3)$ & $125(29,6)$ & 0,004 \\
\hline Gravidez & $73(14,5)$ & $17(20,7)$ & $56(13,2)$ & 0,087 \\
\hline Morte de alguém da família/amigo & $239(47,3)$ & $34(41,5)$ & $205(48,5)$ & 0,277 \\
\hline \multicolumn{5}{|c|}{ Mudança de hábitos } \\
\hline Mudança no hábito de dormir & $166(32,9)$ & $37(45,1)$ & $129(30,5)$ & 0,014 \\
\hline $\begin{array}{l}\text { Mudança de atividade religiosa } \\
\text { Violência sofrida e/ou praticada }\end{array}$ & $54(10,7)$ & $14(17,1)$ & $40(9,5)$ & 0,050 \\
\hline Praticou algum ato de violência & $55(10,9)$ & $13(15,8)$ & $42(9,9)$ & 0,122 \\
\hline Sofreu algum tipo de violência & $69(13,7)$ & $12(14,6)$ & $57(13,5)$ & 0,729 \\
\hline Sofreu algum tipo de abuso sexual & $5(1,0)$ & $3(3,7)$ & $2(0,5)$ & 0,032 \\
\hline Fez sexo por dinheiro & $35(6,9)$ & $14(17,1)$ & $21(5,0)$ & 0,000 \\
\hline \multicolumn{5}{|l|}{ Reabilitação e problemas com justiça } \\
\hline Internou em clínica de reabilitação & $24(4,7)$ & $5(6,1)$ & $19(4,5)$ & 0,568 \\
\hline $\begin{array}{l}\text { Prisão/problemas com a justiça } \\
\text { Trabalho, estudos e renda }\end{array}$ & \multicolumn{3}{|c|}{ Trabalho, estudos e renda } & 0,621 \\
\hline Perda de trabalho & $96(19,0)$ & $17(20,7)$ & $79(18,7)$ & 0,647 \\
\hline Mudança de trabalho & $139(27,5)$ & $24(29,3)$ & $115(27,2)$ & 0,687 \\
\hline Início ou abandonou os estudos & $62(12,3)$ & $10(12,2)$ & $52(12,3)$ & 1,000 \\
\hline Mudança na condição financeira & $200(39,6)$ & $34(41,5)$ & $166(39,2)$ & 0,713 \\
\hline
\end{tabular}

Nota *Somente as questões afirmativas;aTeste Qui-quadrado Exato de Fischer.

Os diferenciais por gênero revelaram que na dimensão Família e Relacionamentos, as usuárias quando comparadas aos usuários, referiram mais frequentemente estes eventos, exceto para morte de alguém na família/amigo $(41,5 \%$ vs $48,5 \%)$. Foram verificadas diferenças proporcionais estatisticamente significantes quanto a perda de filho para o conselho tutelar $(6,1 \%$ vs $1,9 \%)$ e nascimento de criança na família ( $46,3 \%$ vs $29,6 \%)$ ( $p$-valor $\leq 0,05)$.

$\mathrm{Na}$ dimensão Mudanças de hábitos foram verificadas diferenças proporcionais estatisticamente significantes por gênero ( $\mathrm{p}$-valor $\leq 0,05)$, as usuárias quando comparadas aos usuários referiram mais frequentemente mudanças no hábito de dormir ( $45,1 \%$ vs $30,5 \%$ ) e de atividade religiosa ( $17,1 \%$ vs $9,5 \%$ ).

Quanto à dimensão Violência sofrida, as usuárias apresentaram maiores frequências no evento e expressaram também maior vulnerabilidade quando comparadas aos usuários. Foram verificadas diferenças proporcionais estatisticamente significantes quanto aos eventos ter sofrido algum tipo de abuso sexual $(3,7 \%$ vs $0,5 \%)$ e a prática de sexo por dinheiro $(17,1 \%$ vs $5,0 \%)$ (p-valor $\leq 0,05)$. 
Quanto à dimensão Reabilitação e problemas com justiça, os grupos mostraram-se proporcionalmente homogêneos (valor-p $>0,05)$, entretanto as usuárias apresentaram maior frequência quanto a reabilitação $(6,1 \%$ vs $4,5 \%)$ e os usuários maior frequência quanto a problemas com justiça ( $13,4 \%$ vs $16,1 \%)$.

Nas dimensões trabalho, estudos e renda verificou-se homogeneidade quanto a distribuição proporcional dos eventos por gênero ( $p$-valor $>0,05$ ). Entretanto, as usuárias apresentaram frequências superiores a amostra geral quanto aos eventos perda e mudança de trabalho e de condição financeira e igualdade proporcional quanto a iniciar ou abandonar os estudos.

Na Tabela 3, avaliou-se a magnitude da ocorrência (RP) dos eventos de vida que se mostraram associados ao gênero. Destacaram-se as mulheres como mais prevalente em todos os eventos. Na RP1, as chances de ocorrência obtidas, o gênero masculino representou categoria de risco e o feminino referente (ou de comparação). As medidas obtidas revelaram que o gênero masculino atuou como fator protetor na ocorrência de todos os eventos (RP $<1)$, as medidas se mostraram associadas e estatisticamente significantes. Entretanto, ao obtermos a RP2, o gênero feminino representou categoria de risco e o masculino como referente (ou de comparação), verificaram-se associações positivas e estatisticamente significantes, resultou que o gênero feminino atuou como fator de risco a ocorrência de todos os eventos $(\mathrm{RP}>1)$.

Tabela 3. Prevalência, Razão de Prevalência (RP1 e RP2) e respectivos intervalos de confiança a 95\% dos eventos ocorridos nos últimos anos associados ao gênero dos usuários de crack, álcool e outras drogas. Pelotas RS, Brasil, 2011 2012. $(n=505)$

\begin{tabular}{|c|c|c|c|c|c|}
\hline Eventos ocorridos no último ano & Prevalência n (\%) & $\mathrm{RPa}_{1}$ & IC95\% a & $\mathrm{RPa}_{2}$ & IC95\% ${ }^{a}$ \\
\hline \multicolumn{6}{|l|}{ Perda de filho para o conselho } \\
\hline Feminino & $5(6,1)$ & & $1,0 \mathrm{~b}$ & 3,37 & $(1,07-10,57)$ \\
\hline Masculino & $8(1,2)$ & 0,30 & $(0,09-0,93)$ & & $1,0 \mathrm{~b}$ \\
\hline \multicolumn{6}{|l|}{ Nascimento de criança na família } \\
\hline Feminino & $38(46,3)$ & & $1,0 \mathrm{~b}$ & 2,06 & $(1,27-3,33)$ \\
\hline Masculino & $125(29,6)$ & 0,48 & $(0,00-0,79)$ & & $1,0 \mathrm{~b}$ \\
\hline \multicolumn{6}{|l|}{ Mudança de atividade religiosa } \\
\hline Feminino & $14(17,1)$ & & $1,0 \mathrm{~b}$ & 2,00 & $(1,02-3,82)$ \\
\hline Masculino & $40(9,5)$ & 0,51 & $(0,26-0,98)$ & & $1,0 \mathrm{~b}$ \\
\hline \multicolumn{6}{|l|}{ Mudança no hábito de dormir } \\
\hline Feminino & $37(45,1)$ & & $1,0 \mathrm{~b}$ & 1,87 & $(1,16-3,03)$ \\
\hline Masculino & $129(30,5)$ & 0,53 & $(0,33-0,86)$ & & $1,0 \mathrm{~b}$ \\
\hline \multicolumn{6}{|l|}{ Sofreu algum tipo de abuso sexual } \\
\hline Feminino & $3(3,7)$ & & $1,0 \mathrm{~b}$ & 3,94 & $(1,91-8,12)$ \\
\hline Masculino & $2(0,5)$ & 0,12 & $(0,02-0,76)$ & & $1,0 \mathrm{~b}$ \\
\hline \multicolumn{6}{|l|}{ Fez sexo por dinheiro } \\
\hline Feminino & $14(17,1)$ & & $1,0 \mathrm{~b}$ & 8,00 & $(1,31-48,61)$ \\
\hline Masculino & $21(5,0)$ & 0,25 & $(0,12-0,52)$ & & $1,0 b$ \\
\hline
\end{tabular}

Nota: aEstimados em função do odds ratio pelo método verossimilhança da Regressão Logística; bcategoria

As chances de ocorrência do evento perda de filho para o conselho tutelar foi 3,37 vezes superior (IC95\%: 1,07 $-10,57)$ para as mulheres quando comparadas aos homens. Já as chances de nascimento de criança na família fo 2,06 vezes superior (IC95\%: 1,27 - 3,33) para as mulheres quando comparadas aos homens. O evento mudança de atividade religiosa apresentou uma chance de ocorrência 2 vezes superior (IC95\%: 1,02 - 3,82) para as mulheres quando comparadas aos homens. 0 evento mudança no hábito de dormir apresentou uma chance de ocorrência entre as mulheres de 1,87 vezes (IC95\%: 1,16 - 3,03) quando comparadas aos homens. 0 evento abuso sexual apresentou maiores chances de ocorrência entre as mulheres, foi 3,94 vezes superior (IC95\%: 1,91 - 8,12) quando comparadas aos homens. A prática de sexo por dinheiro a chance de ocorrência entre as mulheres foi 8 vezes superior (IC95\%: 1,31 $48,61)$ quando comparadas aos homens.

Discussão

O presente estudo objetivou caracterizar o contexto de vida e a vulnerabilidade de homens e mulheres assistidos pelos serviços de CAPS AD e Redução de Danos de um município do extremo sul do Brasil na perspectiva do gênero.

O gênero masculino foi predominante e representou $83,8 \%$ do total e o feminino $16,2 \%$ evidenciando uma proporção masculina veementemente maior. Estudos epidemiológicos em diferentes países têm evidenciado que a taxa de consumo de drogas é mais elevada entre os homens, contudo, vem sendo registrada uma diminuição da diferença da proporção entre homens e mulheres em se tratando do uso de drogas de um modo geral.6,7

E importante considerar nas usuárias 0 entrecruzamento de comportamentos e vivências subjetivadas ligadas questões como sexualidade, fidelidade, preconceitos, liberdade e morte, imbuídos de desigualdades de gênero e de poder.8

As mudanças de hábitos, especialmente em relação ao sono podem ser explicadas pelo fato do pensamento dos usuários focarem-se no consumo de crack de forma que sono, alimentação, afeto, senso de responsabilidade e sobrevivência perdem o significado.9

A aderência a práticas religiosas pode favorecer a diminuição do consumo, quando esse é desejado, pois ao se envolver em tais práticas, as pessoas agregam um conjunto de valores, símbolos e práticas sociais, que influenciam seus pensamentos e comportamentos acerca da aceitação ou recusa do consumo de drogas. 10

0 percentual de usuárias que sofreram abuso sexual encontra-se menor no presente estudo comparando-se com pesquisa nacional entre usuárias de crack que chegou a índices de até $44 \% .11$ A violência sexual é fenômeno universal que atinge, indistintamente, todas as classes sociais, etnias, religiões e culturas, e sua verdadeira incidência é desconhecida, além disso, a incidência de maus tratos verbais e físicos e de abuso sexual no interior das residências são considerados fatores desencadeantes para o uso de drogas. 12

O fato de a família esconder e negar o uso abusivo de substâncias psicoativas por parte das mulheres pode retardar a busca de tratamento para os agravos decorrentes do consumo da substância e predispor a usuária a situações de violências de ordem familiar.13

Em virtude da fissura que gera sensação de urgência por crack, o usuário pode esgotar rapidamente seus recursos financeiros, impelindo-o a participar de atividades como a prática de sexo pela droga ou por dinheiro para obtê-la. Percebe-se que o estudo demonstra percentual significativamente maior em mulheres de prática de sexo por dinheiro, 0 que pode estar relacionado ao fato dos homens negarem essa prática por acreditarem que interfira na sua masculinidade.

A relação dos gêneros masculino e feminino repercute na maneira de perceber as condutas de homens e mulheres em relação à redução de danos e riscos. Mulheres usuárias de crack são rotuladas como irresponsáveis, prostitutas e criminosas, estando longe de comportamentos socialmente esperados para o gênero feminino.14 Existe estigma e preconceito em torno do consumo de drogas até mesmo por parte de pessoas usuárias de drogas, mais especificamente, do grupo de homens.13

O perfil das mulheres entrevistadas demonstrou ser diferente das pesquisadas no município de São Paulo, em 2008, quando foi relatado que metade das mulheres entrevistadas já teria se prostituído em troca de crack. 0 mesmo estudo ainda identificou a prostituição compulsória, em que homens "emprestam" suas esposas a traficantes ou a outros usuários em troca de crack, de tal forma que o período e o número de pedras são combinados no momento da negociação. 9

A prática de sexo por dinheiro pode aumentar a vulnerabilidade das mulheres, especialmente em relação ao risco de contração de Infecções Sexualmente Transmissiveis (IST's). HIV e AIDS, risco de reincidência de vitimização e degradação física, e ainda uma vez iniciada esta prática pode construir um ciclo vicioso, uma vez que as mulheres podem procurar na droga um apoio.8,13,15

Em relação ao maior número de mulheres procurarem internação para reabilitação pode estar relacionado à maior 
procura por mulheres pelos serviços de saúde em geral. As características sociais da população estudada demonstram que pessoas com maior comprometimento social possuem maior dificuldade de acesso às redes de saúde, tendo maior necessidade de atenção em estratégias de saúde da família e programa de redução de danos.

A fissura pelo crack pode impelir o usuário a participar de atividades ilícitas, o que tem conferido a essa população uma condição de marginalidade e criminalidade, as quais têm sido amplamente divulgadas na mídia, fazendo com que o fenômeno do consumo tenha um impacto maior e seja permeado de estigma na concepção da população em geral.8

O maior envolvimento por parte dos homens com situações de conflito com a lei está descrito na literatura, a qual aponta que em geral as mulheres tendem a valer-se do corpo como moeda de troca, enquanto os homens mais comumente estão ligados ao narcotráfico, porém de forma discreta, há registros do envolvimento de mulheres com o tráfico na função de "mulas".13

O consumo de drogas é fenômeno multifatorial, envolve questões individuais, da droga em si, do contexto socia e cultural. $O$ início do consumo de drogas ilícitas dá-se habitualmente na adolescência, dentro de um cenário socia muitas vezes conturbado, podendo prejudicar a vida escolar e determinar 0 abandono dos estudos. 0 grupo de mulheres estudado foi muito semelhante ao que a literatura tem descrito sobre usuários de drogas: jovens, em fase economicamente ativa e reprodutiva, sem vínculo empregatício e com baixo poder aquisitivo e com nível de escolaridade incompatível com a idade. 12

A dimensão do trabalho pode tornar-se prejudicada devido à baixa escolaridade, perda de vínculos com a família e o meio social, mudanças na aparência, devido à falta de cuidados com o corpo e à alimentação, ainda problemas com pontualidade e assiduidade relacionados ao uso compulsivo de substâncias psicoativas. 10

O número significativamente maior de mulheres que perderam seus filhos para o conselho tutelar pode relacionarse ao fato dos cuidados com as crianças serem tradicionalmente função feminina, e estarem os filhos sob a tutela unicamente das mães. Quando a mulher é usuária de drogas e não vivencia o processo da maternidade esperado para uma mãe, passa a ser taxada pela sociedade, como egoísta e irresponsável para com os filhos, se constituindo numa preocupação social.

O medo de perderem seus filhos para o conselho tutelar, devido a desestrutura de suas vidas, foi um dos principais motivos relatados em estudos recentes para o abandono do uso de substâncias. As mulheres que consomem crack são duplamente estigmatizadas e acabam se sentindo culpadas pelo vício, ocasionando baixa autoestima e sintomas de ansiedade. 10

A maternidade, conforme estudo supracitado, juntamente com o afastamento das áreas de risco e a prática religiosa, são motivações para o abandono das drogas e as que não conseguem abandonar o uso durante a gestação apresentam sentimentos de culpa e baixa autoestima. 9 Estudo aponta que mais da metade das usuárias engravidaram ao menos uma vez desde o início do uso do crack/similares, configurando riscos na gestação e no desenvolvimento neurológico e intelectual das crianças expostas ao uso da droga.14

0 número de nascimentos de crianças significativamente maior entre as mulheres pode estar associado com o número também elevado de separações e divórcios, os quais podem estar relacionados à desvalorização e preconceito exercido por parte dos companheiros com as usuárias de drogas. Ainda existe a questão de não adotarem o uso de preservativo, independentemente de estarem ou não sob efeito de drogas, e sim porque apesar de valorizarem 0 preservativo como mecanismo de prevenção apresentam resistências pessoais ou dos parceiros.13

Considerações Finais

O presente estudo demonstrou que as mulheres usuárias de drogas, ainda que em menor número do que os homens apresentaram maior ocorrência de violência sofrida e abuso sexual, bem como perda de seus filhos para 0 conselho tutelar e mudanças em seus hábitos de sono. Ao mesmo tempo, buscaram mais frequentemente a reabilitação. A mulher usuária de drogas apresenta-se mais vulnerável aos riscos, perdas e ao preconceito.

O contexto dos homens usuários, por sua vez, reflete uma realidade de baixa escolaridade e envolvimento com problemas judiciais, ressaltando assim, a situação de vulnerabilidade em que os usuários se apresentavam. Contudo, chama a atenção que, tanto homens como mulheres, em sua ampla maioria, desempenham algum tipo de atividade remunerada. Apesar da baixa renda encontrada, os usuários não deixavam de trabalhar, de modo formal ou informal. Esses achados sublinham a necessidade da criação e execução de políticas públicas que considerem a pessoa na sua singularidade, de acordo com suas características e vulnerabilidades. Políticas que ofereçam programas

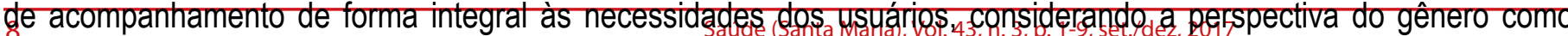

REFERÊNCIAS

1. Lima, HE, Nogueira, MJ, Allanic, LAG, Nogueira, C. Adolescência e Saúde: Indicadores do uso de álcool e outras dROgas APARTIR DE UM ESTUDO MULTICÉNTRICO. REVISTA TECER. 2015; 8(14):1-12.

2. Bastos FI, Bertoni N (orgs). Perfil dos usuários de Crack e/ou similares no Brasil. Rio de Janeiro: ICICT/FIOCRUZ; 2014.

3. Rodrigues LSA, Sena ES, Silva dM, Carvalho PAL, Amorim CR. Perfil dos Usuários Atendidos em um Centro de Atenção Psicossocial - Álcool e Drogas. Journal of NuRsing UFPE. 2013;7: 5191-7.

4. Brasill. Ministério da Justiça. Secretaria Nacional de Políticas sobre Drogas. Prevenção do uso de drogas: capacitação PARA CONSELHEIROS E LIDERANÇAS COMUNITÁRIAS. 5.ED.-BRASILIA: SENAD,2013. 450P.

5. Macagnan, JP, Menetrier, JV, Bortoloti, DS. Perfil dos usuários de um centro de atenção psicossocial No Municipio de Franscisco Beltrão- Paraná. Revista Biosaúde. 2014;16:2.

6. Silva LO. Mulher, drogas e questöes de gênero. Instituto de Psicologia da Universidade de Brasilia. Especialização em Saúde Mental, Álcool e Outras Drogas [Monografia]. Brasílaa, 2015.

7. Leigh WA, Li Y (orgs). Women of Color Health Data Book. New York, USA: National Institutes of Health; 2014

8. Ramiro FS, PadoVANI RC, TuCCI AM. CONSUMO DE CRACK A PARTIR DAS PERSPECTIVAS DE GÊNERO E VULNERABLIIDADE: UMA REVISÃO SOBRE O FENÔMENO. SAÚdE EM DEBATE. 2014; 38(101):379-92.

9. Botti, NCL, Machado, JSA, Tameirão, FV. Perfll sociodemográfico e padrão do uso de CRACK Entre usuárIos em tRatamento no Centro de Atenção Psicossocial. Rev Estudos e Pesquisas em Psicologia. 2014; 14(1).

10. Oliveira MM, Kantorski LP, Coimbra VCC, Ferreira RZ, Ferreira GB, Cruz VD. Consequências relacionadas ao consumo DE CRACK ENTRE MULHERES E MOTIVAÇ̄ES PARA O ABANDONO DA DROGA. SMAD. 2014; 10(3):119-25.

11. Souza MCH, Mühten BKV, Coelho LRM, Oliveira CP, Rodrigues VS, Oliveira MS. Assertividade em mulheres dependentes DE CRACK. ALETHEIA. 2014; 43(44):105-115.

12. Marangoni SR, Oliveira MLF. Fatores desencadeantes do uso de drogas de abuso em mulheres. Texto Contexto Enferm. 2013; 22(3):662-70

13. RamiRo FS, PADoVAnI RC, TucCI AM. Consumo de CRACK A PARTIR DAS PERSPECTIVAS DE GÊNERO E VULNERABILIDADE: UMA REVISÃO Sobre o FenÔMeno. SaÚde Debate. 2014; 38(101):379-92.

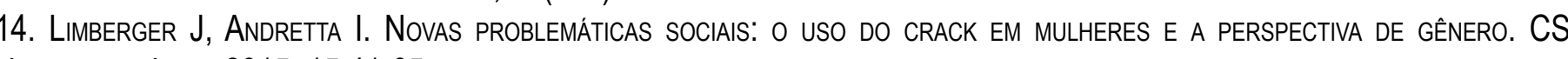
UNIVERSIDAD ICESI. 2015; 15:41-65.

15. Cruz VD, Oliveira MM, Pinho LB, Coimbra VCC, Kantorski LP, Oliveira JF. Sociodemographic Conditions and patterns of CRACK USE AMONg WOMEn. TeXto ConteXto Enferm. 2014; 23(4):1068-76. 